\title{
Chemical evolution and depletion pattern in Damped Lyman $\alpha$ systems
}

\author{
J. L. Hou ${ }^{1,2,3,4}$, S. Boissier ${ }^{2,5}$, and N. Prantzos ${ }^{2}$ \\ 1 Shanghai Astronomical Observatory, CAS, Shanghai 200030, PR China \\ e-mail: hjlyx@center.shao.ac.cn \\ 2 Institut d'Astrophysique de Paris, 98bis, Bd. Arago, 75014 Paris, France \\ 3 National Astronomical Observatories, CAS, PR China \\ 4 Joint Lab. of Optical Astronomy, CAS, PR China \\ 5 Institute of Astronomy, University of Cambridge, Madingley Road, Cambridge, CB3 0HA, UK \\ e-mail: boissier@ast.cam.ac.uk
}

Received 18 October 2000 / Accepted 7 February 2001

\begin{abstract}
Dust depletion plays a key role in understanding the nature of Damped Lyman $\alpha$ systems (DLAs). In this paper we point out a previously unnoticed anticorrelation between the observed abundance ratio $[\mathrm{X} / \mathrm{Zn}]$ (where $\mathrm{Zn}$ is assumed to be undepleted and $\mathrm{X}$ stands for the refractories $\mathrm{Fe}, \mathrm{Cr}$ and $\mathrm{Ni}$ ) and metal column density $\left([\mathrm{Zn} / \mathrm{H}]+\log \left(N_{\mathrm{HI}}\right)\right)$ in DLAs. We suggest that this trend is an unambiguous sign of dust depletion, since metal column density is a measure of the amount of dust along the line of sight. Assuming that DLAs are (proto-) galactic disks and using detailed chemical evolution models with metallicity dependent yields we study chemical evolution and dust depletion patterns for $\alpha$ and iron-peak elements in DLAs. When observational constraints on the metal column density of DLAs are taken into account (as suggested in Boissé et al. 1998) we find that our models reproduce fairly well the observed mild redshift evolution of the abundances of 8 elements $(\mathrm{Al}, \mathrm{Si}, \mathrm{S}$, $\mathrm{Cr}, \mathrm{Mn}, \mathrm{Fe}, \mathrm{Zn}$ and $\mathrm{Ni}$ ) as well as the observed scatter at a given redshift. By considering the aforementioned dependence of abundance ratios on metal column density, we further explore the general dust depletion pattern in DLAs, comparing to our model results and to a solar reference pattern. We find that for low metal column densities (no depletion), our models compare fairly well to the data, while a solar pattern has difficulties with Mn. At high metal column densities (amount of depletion $\sim 0.5 \mathrm{dex}$ ), the solar pattern describes the data quite well, while our models have difficulties with S. We suggest that further measurements of those key elements, i.e. $\mathrm{Zn}, \mathrm{S}$ and Mn, will help us to gain more insight into the nature of DLAs. The presently uncertain nucleosynthesis of $\mathrm{Zn}$ in massive stars (on which a large part of these conclusions is based) should be carefully scrutinised.
\end{abstract}

Key words. ISM: abundances - ISM: dust, extinction - galaxies: spiral - galaxies: abundances - galaxies: evolution

\section{Introduction}

Damped Lyman $\alpha$ systems (DLAs) are high column density gaseous systems $\left(N_{\mathrm{HI}}>210^{20} \mathrm{~cm}^{-2}\right)$, detected through their absorption lines in the optical spectra of quasars, up to relatively high redshifts (up to $z \sim 5$ ). Their study constitutes a powerful means to investigate the properties of distant galaxies (or of their building blocks). In particular, DLA metal abundances have been widely used in the past few years to probe the nature of DLAs (e.g. Lauroesch et al. 1996; Lu et al. 1996; Pettini et al. 1994, 1997b, 1999, 2000; Prochaska \& Wolfe 1999, 2000 , and many others). However, it is not clear whether the observed abundances allow that, because of various

Send offprint requests to: N. Prantzos,

e-mail: prantzos@iap.fr biases: depletion of metals into dust (Pei \& Fall 1995) or bias against too high or too low metal column densities (Boissé et al. 1998). The latter bias, in particular, may explain the observed absence of evolution in the absolute abundance of $\mathrm{Zn} / \mathrm{H}$ as a function of redshift, as suggested in Prantzos \& Boissier (2000a, hereafter PB2000a).

Abundance ratios offer a better diagnostic tool than absolute abundances for the study of the chemical evolution of a system. However, in the case of DLAs, the possibility of depletion into dust grains (even to a small extent) makes difficult a direct interpretation of the observed abundance patterns. Several attempts have been made in recent years to recover the intrinsic abundance pattern from the observed one and to compare the result to well-known patterns of stars in the Milky Way (e.g. 
Vladilo 1998, 1999; Pettini et al. 2000), but with rather modest success up to now.

In this work, we reassess the question of abundance patterns in DLAs. First, we point out an anticorrelation between the observed abundance ratio $\mathrm{X} / \mathrm{Zn}$ (where $\mathrm{Zn}$ is assumed to be undepleted and $\mathrm{X}$ stands for the refractories $\mathrm{Fe}, \mathrm{Cr}$ and $\mathrm{Ni}$ ) and metal column density (Sect. 2). We suggest that this, previously unnoticed, trend is an unambiguous sign of depletion, since metal column density is a measure of the amount of dust along the line of sight. We then study the chemical evolution of those systems, assuming they are (proto-) galactic disks. This assumption is rather controversial, since other systems (e.g. Low Surface Brightness galaxies, dwarf irregulars, galactic haloes; see Jimenez et al. 1998; Matteucci et al. 1997; Valageas et al. 1999; Haehnelt et al. 1998; Petitjean \& Ledoux 1999) have also been suggested; however, the various zones of our multi-zone disk models have sufficiently diverse chemical histories to account for systems other than the Milky Way, a "prototype" disk galaxy. Our models, briefly presented in Sect. 3.1, have been shown to reproduce successfully a large number of observed properties of spirals at high and low redshifts. An important ingredient of our study is the use of metallicity-dependent yields for massive stars and SNIa (presented in Sect. 3.2). In Sect. 4 we show that our results reproduce fairly well both the observed slow evolution with redshift of the absolute abundances of several elements, and the corresponding scatter; observational biases, as already suggested in PB2000a for the case of $\mathrm{Zn}$, is a key to that success. In Sect. 5 we compare our results to observed abundance ratios in DLAs, taking into account the aforementioned trend of depletion vs. metal column density. We find that for low metal column densities (i.e. no depletion) our models compare fairly well to the data, while a solar pattern has difficulties with Mn. At high column densities (small depletion) the solar pattern describes the data quite well, while our models have difficulties with $\mathrm{S}$. We suggest that further measurements of those key elements, i.e. Zn, S and Mn, will help to gain some insight into the nature of DLAs. In any case, the presently uncertain nucleosynthesis of $\mathrm{Zn}$ (on which a large part of these conclusions is based) should be carefully scrutinised.

\section{DLA abundance ratios vs. metal column density}

Studies of the elemental abundances of the interstellar medium (ISM) in the Milky Way have shown that the gas phase abundances of most elements are below the corresponding solar values. This underabundance is generally attributed to depletion into dust grains. The amount of depletion depends on the nature of the interstellar medium. In cold, dense, disk gas, $\mathrm{Mn}, \mathrm{Cr}, \mathrm{Fe}$ and $\mathrm{Ni}$ are considerably depleted (by 1.5 to 2 dex), while $\mathrm{S} / \mathrm{H}$ is nearly solar (Savage \& Sembach 1996 and references therein). In warm disk gas, the amount of depletion is smaller $(\sim 1$ dex for $\mathrm{Fe}$-peak elements), while in warm, halo gas, a negligible amount of depletion is found ( $<0.5$ dex) for all elements, except $\mathrm{Fe}$ and $\mathrm{Ni}$ which are depleted by 0.6 to 0.8 dex.
A more quantitative assessement of the amount of depletion can be made by plotting the abundance of each ionic species as a function of the hydrogen column density $N_{\mathrm{HI}}$ along the line of sight. In a recent work, Wakker \& Mathis (2000) compiled data from several studies concerning high column densities $\left(N_{\mathrm{HI}}=10^{20}\right.$ to $\left.710^{21} \mathrm{~cm}^{-2}\right)$ and extended that range down to $10^{18} \mathrm{~cm}^{-2}$ by using data from high - and intermediate-velocity gas clouds (most of them have intrinsically near-solar abundance). They showed that over the whole range of HI column densities (covering almost three orders of magnitude), there is a tight anticorrelation between $\log (\mathrm{X} / \mathrm{H})$ and $\log \left(N_{\mathrm{HI}}\right)$ for several elements: high column densities correspond to a larger amount of depletion, with a remarkably low dispersion around each value of $N_{\mathrm{HI}}$. The slope of the anticorrelation depends on the ionic species considered; it is $-0.39 \pm 0.04$ for MnII and $-0.59 \pm 0.04$ for FeII, two species that have also been measured in DLAs.

It is interesting to see whether such an anticorrelation exists for DLAs. In Table 1 we present a compilation of currently available data for DLA abundances that we use in this work. The redshift range of the DLAs and their number in each survey are also given in the Table. Data displayed in all our figures are directly taken from the source references, with no attempt to homogenize them.

Table 1. $\alpha$ and Fe-peak element abundances in DLAs

\begin{tabular}{|c|c|c|c|c|c|c|c|c|c|c|}
\hline Ref. & $z_{\mathrm{abs}}$ & obj. & $\mathrm{Al}$ & $\mathrm{Si}$ & $\mathrm{S}$ & $\mathrm{Cr}$ & $\mathrm{Mn}$ & $\mathrm{Fe}$ & $\mathrm{Ni}$ & $\mathrm{Zn}$ \\
\hline (1) & $0.3-1.0$ & 6 & & & & & & & & $\mathrm{X}$ \\
\hline$(2)$ & $1.7-4.4$ & 15 & & $\mathrm{X}$ & $\mathrm{X}$ & & & $\mathrm{X}$ & & $\mathrm{X}$ \\
\hline (3) & $1.9-2.5$ & 6 & & $\mathrm{X}$ & $\mathrm{X}$ & $\mathrm{X}$ & & $\mathrm{X}$ & & $\mathrm{X}$ \\
\hline (4) & $0.68,1.15$ & 2 & & $\mathrm{X}$ & & & $\mathrm{X}$ & $\mathrm{X}$ & & $\mathrm{X}$ \\
\hline (5) & $0.7-4.5$ & 23 & $\mathrm{X}$ & $\mathrm{X}$ & $\mathrm{X}$ & $\mathrm{X}$ & $\mathrm{X}$ & $\mathrm{X}$ & $\mathrm{X}$ & $\mathrm{X}$ \\
\hline (6) & 2.3090 & 1 & & & $\mathrm{X}$ & & & $\mathrm{X}$ & & \\
\hline (7) & 3.3901 & 1 & & $\mathrm{X}$ & & $\mathrm{X}$ & & $\mathrm{X}$ & $\mathrm{X}$ & $\mathrm{X}$ \\
\hline (8) & $1.7-3.0$ & 15 & & & & $\mathrm{X}$ & & & & $\mathrm{X}$ \\
\hline (9) & $0.7-3.3$ & 17 & & & & $\mathrm{X}$ & & & & $\mathrm{X}$ \\
\hline (10) & $1.1-1.5$ & 5 & & $\mathrm{X}$ & & $\mathrm{X}$ & $\mathrm{X}$ & $\mathrm{X}$ & $\mathrm{X}$ & $\mathrm{X}$ \\
\hline (11) & $0.6-1.5$ & 6 & & $\mathrm{X}$ & & $\mathrm{X}$ & $\mathrm{X}$ & $\mathrm{X}$ & $\mathrm{X}$ & $\mathrm{X}$ \\
\hline (12) & $1.7-4.2$ & 22 & $\mathrm{X}$ & $\mathrm{X}$ & $\mathrm{X}$ & $\mathrm{X}$ & & $\mathrm{X}$ & $\mathrm{X}$ & $\mathrm{X}$ \\
\hline (13) & $2.4-4.2$ & 4 & & & & & & $\mathrm{X}$ & & \\
\hline
\end{tabular}

References: (1) Boissé et al. (1998); (2) Centurion et al. (1998); (3) Centurion et al. (2000); (4) de la Varga et al. (2000); (5) Lu et al. (1996); (6) Molaro et al. (1998); (7) Molaro et al. (2000); (8) Pettini et al. (1994); (9) Pettini et al. (1997b); (10) Pettini et al. (1999); (11) Pettini et al. (2000); (12) Prochaska \& Wolfe (1999); (13) Prochaska \& Wolfe (2000).

In order to evaluate the depletion in DLAs as a function of column density, one cannot use the absolute abundances $[\mathrm{X} / \mathrm{H}]$ because DLAs have different metallicities: a low $[\mathrm{X} / \mathrm{H}]$ value may correspond either to large depletion or to little chemical evolution (or both). For those reasons, we adopt here the $[\mathrm{X} / \mathrm{Zn}]$ ratio as a measure of depletion of a given element $\mathrm{X}$, assuming that $\mathrm{Zn}$ is always undepleted, irrespectively of the HI column density. By using $[\mathrm{X} / \mathrm{Zn}]$ to measure depletion we also assume that the real $[\mathrm{X} / \mathrm{Zn}]$ value of each DLA (i.e. before depletion) is always the same, which is probably not the case. However, we are looking here for systematic effects as a function of 
column density; any deviations of the real $[\mathrm{X} / \mathrm{Zn}]$ from the value adopted here $([\mathrm{X} / \mathrm{Zn}]=0)$ would merely manifest as dispersion around an average trend.

On the left panels of Fig. 1 we present the DLA data of [X/Zn] as a function of $N_{\mathrm{HI}}$ column density. No anticorrelation is found, contrary to the situation in gas clouds in our Galaxy, which is shown by solid lines in the panels of $\mathrm{Mn}$ and Fe (from the fits of Wakker \& Mathis 2000). The absence of such an anticorrelation in DLAs, compared to local measurements, seems puzzling. Does it mean that depletion (if any) in DLAs is independent of HI column density?

Here, we suggest that a proper measurement of depletion is provided not by the HI column density, but rather by the column density of (undepleted) metals, e.g. by $\log \left(N_{\mathrm{Zn}}\right)=[\mathrm{Zn} / \mathrm{H}]-7.83+\log \left(N_{\mathrm{HI}}\right)$, where $-7.83=$ $\log (\mathrm{Zn} / \mathrm{H})_{\odot}$. In the case of clouds in our Galaxy, metallicity is always the same $([\mathrm{Zn} / \mathrm{H}] \sim 0)$, making it possible to use $N_{\mathrm{HI}}$ instead of $N_{\mathrm{Zn}}$. However, in DLAs, because of the different $[\mathrm{Zn} / \mathrm{H}], N_{\mathrm{HI}}$ is not proportional to $N_{\mathrm{Zn}}$ and cannot be used to measure depletion.

When $[\mathrm{X} / \mathrm{Zn}]$ is plotted as a function of $\mathcal{F}=[\mathrm{Zn} / \mathrm{H}]+$ $\log \left(N_{\mathrm{HI}}\right)$ (right panels in Fig. 1), one can see that: i) there is no dependence of $\mathrm{Si} / \mathrm{Zn}$ and $\mathrm{S} / \mathrm{Zn}$ on $\mathcal{F}$, as expected for those two elements, which are also found not to be substantially depleted in local gas clouds, and ii) there is a clear anticorrelation between $[\mathrm{X} / \mathrm{Zn}]$ and $\mathcal{F}$ for the refractory elements $\mathrm{Cr}, \mathrm{Fe}$ and $\mathrm{Ni}$ (as can be seen from the dashed lines) while the scarce data for $\mathrm{Mn}$ do not allow for a conclusion.

We think that the anticorrelation obtained between $[\mathrm{X} / \mathrm{Zn}]$ and $\mathcal{F}$ in DLAs is an unmistakable sign of depletion for $\mathrm{Fe}, \mathrm{Cr}$ and $\mathrm{Ni}$. Metal column density is the appropriate factor, since it measures the total amount of metal along the line of sight, and thus the amount of dust; $N_{\mathrm{HI}}$ alone is inappropriate, since further assumptions about the dust to gas ratio have to be made.

How can one explain the fact that $[\mathrm{X} / \mathrm{Zn}]$ seems to be independent of $N_{\mathrm{HI}}$ for all elements in DLAs? We note that there exists a well known anticorrelation between metallicity, as expressed by $[\mathrm{Zn} / \mathrm{H}]$, and $\mathrm{HI}$ column density in DLAs. This anticorrelation, first noticed by Boissé et al. (1998), is attributed to selection biases (since Zn is not depleted into dust grains): regions of high $[\mathrm{Zn} / \mathrm{H}]$ and high $N_{\mathrm{HI}}$ are opaque to light from background quasars and thus undetectable, while regions of low $[\mathrm{Zn} / \mathrm{H}]$ and low $N_{\mathrm{HI}}$ (i.e. of low $\mathrm{Zn}$ column density) are not detectable by current DLA surveys. In PB2000a we substantiated this argument by running detailed chemical evolution models for disk galaxies. We showed how this "filter" can lead to a "no-evolution" picture for the average DLA metallicity as a function of redshift, in agreement with observations. We shall return to that question in Sect. 3.3, presenting results for other elements than $\mathrm{Zn}$. Here we simply note that, since high $N_{\mathrm{HI}}$ DLAs are asociated, on average, with low $[\mathrm{Zn} / \mathrm{H}]$ and vice-versa, the depleted ratio $[\mathrm{X} / \mathrm{Zn}]$ is expected to be, on average, independent of $N_{\mathrm{HI}}$, as observed. On the contrary, when $[\mathrm{X} / \mathrm{Zn}]$ is plotted as a function of $\mathcal{F}$ (which is directly proportional to the amount of dust), the anticorrelation expected by local observations becomes clear.

An inspection of the Fe panel in Fig. 1 shows that the absolute $[\mathrm{Fe} / \mathrm{Zn}]$ values are systematically higher in DLAs than in the Milky Way clouds (by $\sim 0.7$ dex); a similar remark also holds for Mn, although the scarcity of data makes its case less compelling. If metal column density is the relevant factor, as argued in the previous paragraphs, how can this systematic offset (by factor of $\sim 5$ ) be understood? We see two possibilities:

1) The undepleted $[\mathrm{Fe} / \mathrm{Zn}]$ in DLAs is not zero (i.e. the $\mathrm{Fe} / \mathrm{Zn}$ ratio is not solar) as assumed here, but $\sim 0.7$ (i.e. 5 times solar); however, despite current uncertainties on the nucleosynthesis of $\mathrm{Zn}$, this possibility is quite implausible. Indeed, $\mathrm{Fe} / \mathrm{Zn}$ is solar on average in both halo and disk stars of the Milky Way.

2) Wakker \& Mathis (2000) suggest that the very small scatter around the average depletion pattern in the Milky Way clouds (within dotted lines in the Fe panels of Fig. 1) is due to the fact that one single cloud dominates the column density of each line of sight. In that case, a single value of (depleted) $\mathrm{Fe} / \mathrm{Zn}$ corresponds to a given value of $N_{\mathrm{HI}}$ (or of $\mathcal{F}$, since $[\mathrm{Zn} / \mathrm{H}] \sim$ solar in the Milky Way clouds). In the case of DLAs, it is tempting to assume that a given line of sight intercepts several clouds: the depletion of $[\mathrm{Fe} / \mathrm{Zn}]$ within each cloud would be small, but the total column density of metals $(\mathcal{F})$ would be high. This picture could explain the offset of DLAs with respect to the local pattern in Fig. 1. Taking into account the error bars in $[\mathrm{Fe} / \mathrm{Zn}]$ values and the possibility of systematic errors, it is difficult to tell whether a couple of clouds or a larger number is necessary.

At this point, we notice that in the case that DLAs are composed by many systems of individual column densities $\log N(\mathrm{HI})<19.5$, ionisation effects could play an important role in shaping the observed abundance ratios. As suggested recently by Howk \& Sembach (1999), the observed ratios may systematically overestimate the intrinsic ones in the cases of $\mathrm{Si} / \mathrm{Fe}$ and $\mathrm{Zn} / \mathrm{Cr}$, if ionised gas is present in these systems, "thereby mimicking the effects of alpha-element enrichment or dust depletion". Izotov et al. (2000) have further explored this issue, on the basis of similarities observed between some DLAs and nearby blue compact dwarf galaxies; using a schematic two-zone model, they found that ionisation effects may play some role in shaping the observed abundances of these systems, although they recognised that their model suffered from various uncertainties related to the unknown structure and geometry of the absorbing systems, the spatial distribution of the ionising sources and their radiation field. We do not consider this possibility any further here, since we think that the anticorrelation displayed in Fig. 1, i.e. the dependence of $\mathrm{X} / \mathrm{Zn}$ on $\mathrm{Zn}$ column density for well known refractory elements (like $\mathrm{Fe}, \mathrm{Ni}$ and $\mathrm{Cr}$ ) but not for e.g. $\mathrm{Si}$, suggests that dust depletion is the main factor.

The observations of Fig. 1 tell us nothing about the real (undepleted) abundances in DLAs; further 


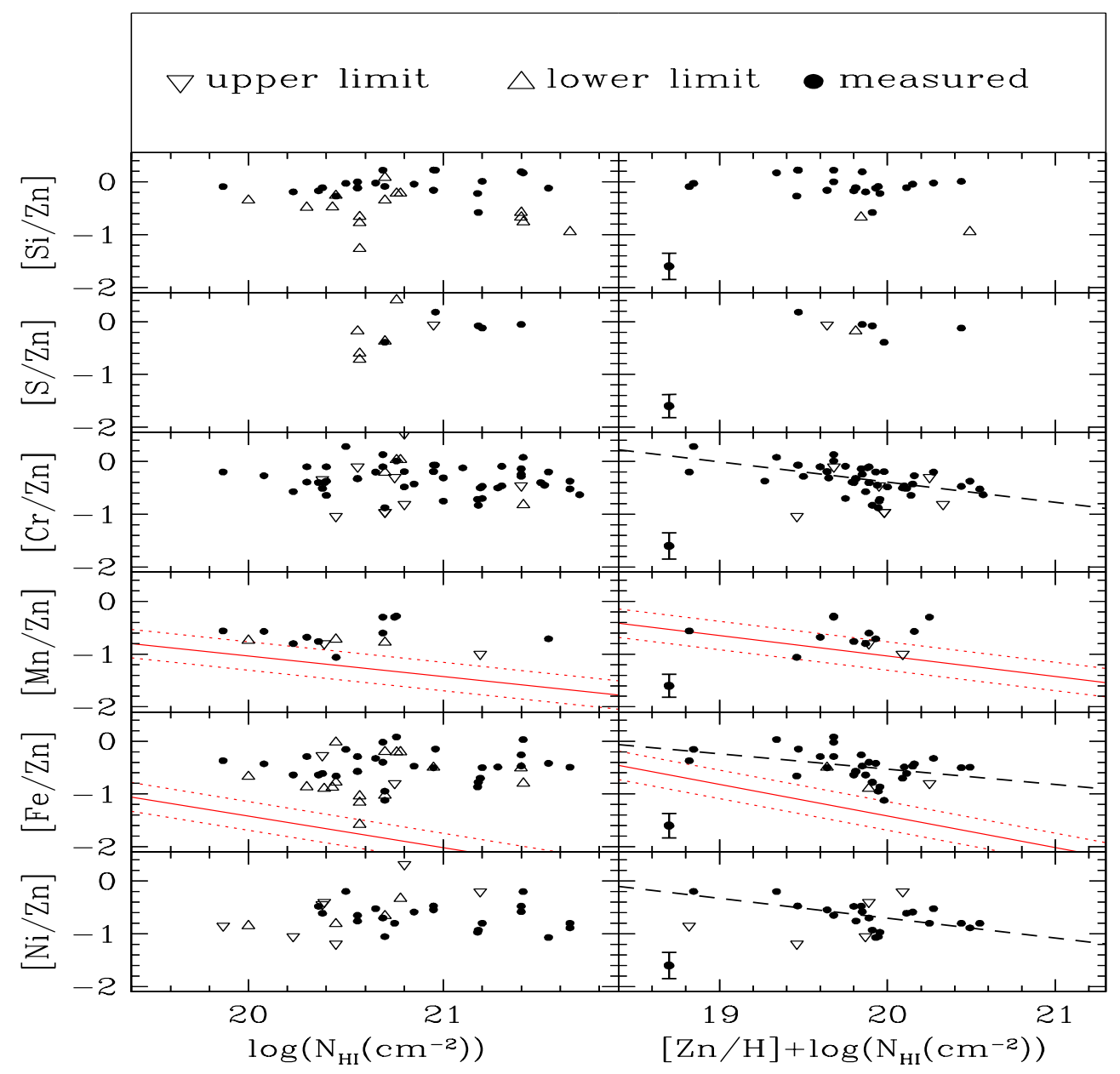

Fig. 1. Gas phase abundance ratios $[\mathrm{X} / \mathrm{Zn}]$ in DLAs, plotted as a function of HI column density (left panels) and of Zn column density (right panels). Data are from references given in Table 1 and corresponding typical uncertainties are shown in each of the right panels by vertical error bars. No correlation is seen in the left panels, while in the right panels, an anticorrelation is found for the refractory elements $\mathrm{Cr}, \mathrm{Fe}$ and $\mathrm{Ni}$ (shown by dashed lines, obtained by a least squares fit to the data). In the panels of $\mathrm{Mn}$ and Fe, solid and dotted lines show the corresponding trends observed in Milky Way clouds and their associated uncertainties (see text for discussion)

assumptions have to be made for that. The investigation of the abundances and the depletion patterns in DLAs by means of a consistent chemical evolution model is the subject of the following sections.

\section{DLAs as disk galaxies}

The details of our model for the chemical evolution of spiral disks are described in Boissier \& Prantzos (2000, 1999; hereafter BP2000 and BP1999, respectively), while its application to the evolution of $\mathrm{Zn}$ in DLAs is made in Prantzos \& Boissier (2000a, hereafter PB2000a). In Sect. 3.1 we briefly recall the main features of the model and its success in reproducing a large number of observations concerning present day disks. In Sect. 3.2 we present in some detail the only two novel ingredients with respect to $\mathrm{BP} 2000$ and $\mathrm{BP} 1999$, namely the metallicitydependent yields of Woosley \& Weaver (1995, hereafter WW1995) for intermediate mass elements, and the new yields of SNIa from of Iwamoto et al. (1999). In Sect. 3.3 we extend our study to the evolution of intermediate mass elements observed in DLAs.

\subsection{Description of the model}

A detailed model of the chemical and spectro-photometric evolution of the Milky Way is constructed in BP1999. The disk of our Galaxy is simulated as an ensemble of concentric rings gradually built up by infall of gas of primordial composition. We adopted a Star Formation Rate (SFR) suggested by the theory of density waves $\psi \propto \Sigma_{\text {gas }}^{1.5} V(R) / R$ (where $V(R)$ is the circular velocity at radius $R$ ), which is 
in agreement with the observed current SFR profile in the Milky Way. The infall rate is exponentially declining, with a timescale $\tau=7 \mathrm{Gyr}$ in the local disk as to reproduce the observed G-dwarf metallicity distribution. Inner galactic zones are formed earlier than the outer disk (formation "inside-out"). The number of observables in our Galaxy, concerning the history of the solar neighborhood as well as the current profiles of various quantities, is larger than the few "free" parameters involved in this model and testifies to its validity.

After "calibrating" the model on the Milky Way (in fact, the SFR efficiency and the infall timescales as a function of local surface densities) we extended it to the study of local spirals (BP2000). We used simple "scaling laws", suggested by Mo et al. (1998) in the framework of Cold Dark Matter scenarios of galaxy formation. Within this simplified approach, disks are characterised by two parameters: circular velocity $V_{\mathrm{C}}$ (determined by the mass of the dark halo), and spin parameter $\lambda$ (measuring the specific angular momentum of the dark halo). For instance, the disk scalelength is $R_{\mathrm{d}}=R_{\mathrm{d}, \mathrm{MW}} *\left(V_{\mathrm{C}} / V_{\mathrm{C}, \mathrm{MW}}\right) *\left(\lambda / \lambda_{\mathrm{MW}}\right)$, where the index MW refers to the corresponding value in the Milky Way (see BP2000 for details).

Our grid of models covers the range $80<$ $V_{\mathrm{C}}\left(\mathrm{km} \mathrm{s}^{-1}\right)<360$ and $1 / 3<\lambda / \lambda_{\mathrm{MW}}<3$, which corresponds to current High Surface Brightness disks. Our results reproduce quite satisfactorily a large number of observed properties of present-day disks: sizes, surface brightness, Tully-Fisher relationship, colours, abundances, integrated spectra, etc. (BP2000). Also, the model reproduces the observed abundance gradients in disk galaxies (Prantzos \& Boissier 2000b) and the gas fraction, star formation efficiency and effective ages of a large sample of nearby spirals (Boissier et al. 20001). We notice that, according to our models, massive disks form quite rapidly (in a few Gyr) while it takes several Gyr for the small ones to be formed.

In order to apply the model to the study of DLAs, the following statistical factors were taken into account:

First, at redshift $z=0$, a velocity distribution function can be obtained by combining a Schechter luminosity function and an observed Tully-Fisher relationship, as in Gonzalez et al. (2000):

$F_{V}(V) \mathrm{d} V=\tilde{\Psi}_{*}\left(\frac{V}{V_{*}}\right)^{\beta} \exp \left[-\left(\frac{V}{V_{*}}\right)^{n}\right] \frac{\mathrm{d} V}{V_{*}}$.

For the parameters of Eq. (1) we adopted the values of the fifth row of Table 4 in Gonzalez et al. (2000), corresponding to the velocity interval covered by our models; another choice would have small impact on the results of this paper.

Results of numerical simulations (see e.g. Mo et al. 1998) give the distribution function of the spin parameter $\lambda$ :

$F_{\lambda}(\lambda) \mathrm{d} \lambda=\frac{1}{\sqrt{2 \pi} \sigma_{\lambda}} \exp \left[-\frac{\ln ^{2}(\lambda / \bar{\lambda})}{2 \sigma_{\lambda}^{2}}\right] \frac{\mathrm{d} \lambda}{\lambda}$

with $\bar{\lambda}=0.05$ and $\sigma_{\lambda}=0.5$.
Finally, the probability that a line of sight to a QSO intercepts a disc in the radius interval $[R, R+\mathrm{d} R]$ is proportional to the geometrical cross-section $F_{R}(R) \mathrm{d} R=$ $2 \pi R \mathrm{~d} R / \pi R_{\mathrm{M}}^{2}$ (where $R_{\mathrm{M}}$ is the radius of the largest disc in our models), favouring the detection of the outer regions of the larger disks.

At any time, or redshift, the average value of a quantity $Q$ (for instance an abundance ratio) in our models is computed from:

$\langle Q\rangle=\frac{\int_{\lambda} \int_{V_{\mathrm{C}}} \int_{R} F \Phi(\mathcal{F}) Q\left(\lambda, V_{\mathrm{C}}, R\right) \mathrm{d} R \mathrm{~d} V_{\mathrm{C}} \mathrm{d} \lambda}{\int_{\lambda} \int_{V_{\mathrm{C}}} \int_{R} F \Phi(\mathcal{F}) \mathrm{d} R \mathrm{~d} V_{\mathrm{C}} \mathrm{d} \lambda}$

where $F=F\left(\lambda, V_{\mathrm{C}}, R\right)=F_{V}\left(V_{\mathrm{C}}\right) F_{\lambda}(\lambda) F_{R}(R)$. We implicitly assume that those three functions are mutually independent and also time-invariable. This is of course an over-simplification, valid as long as mergers and intereactions do not modify $F_{V}$ and $F_{\lambda}$ greatly. This is probably a good approximation at low redshifts, but becomes more and more problematic at high redshifts $(z>3)$.

The function $\Phi$ in Eq. (3) plays the role of a "filter". To obtain the true mean value over the whole ensemble of disks, one should take $\Phi(\mathcal{F})=1$. However, Boissé et al. (1998) argued that observations of DLAs suffer from selection biases, as discussed in Sect. 2. Measurement of zinc abundances vs. HI column density show an anticorrelation, resulting from a bias against too large or too small values of $\mathcal{F}=[\mathrm{Zn} / \mathrm{H}]+\log (N(\mathrm{HI}))$.

To account for that bias, we apply Eq. (3) with $\Phi(\mathcal{F})=1$ for $18.8<\mathcal{F}<21$ and 0 elsewhere; this gives the average value of $Q$ over those parts of our disks that have DLA-like properties (at least, as far as Zn abundances and HI column densities are concerned). As shown in PB2000a, this empirical "filter" can explain the weak evolution with redshift of $[\mathrm{Zn} / \mathrm{H}]$, and the corresponding average value and observed scatter among DLAs (see also top-left pannel in Fig. 2 and discussion in Sect. 3.3). In the present work we extend the model to the study of other elements; the adopted yields are presented in the next section.

\subsection{Yields of massive stars and SNIa}

An important ingredient in studies of galactic chemical evolution is the stellar yields of various elements. Massive stars are the main producers of most heavy isotopes in the Universe. Elements up to $\mathrm{Ca}$ are mostly produced in such stars by hydrostatic burning, whereas iron peak elements are produced by the final supernova explosion (SNII), and by white dwarfs exploding in binary systems as SNIa. Most of $\mathrm{He}, \mathrm{C}, \mathrm{N}$ and minor $\mathrm{CO}$ isotopes, as well as s-nuclei come from intermediate mass $\operatorname{stars}\left(2-8 M_{\odot}\right)$. Since we are mainly concerned with the abundances of $\alpha$ and iron-peak elements in DLAs, we consider no yields from intermediate mass stars in this work.

We use the metallicity dependent yields of WW1995, which are given for stars of mass $M=12,13,15,18$, $20,22,25,30,35$ and $40 M_{\odot}$ and metallicities $Z / Z_{\odot}=0$, $10^{-4}, 10^{-2}, 10^{-1}$ and 1 . The WW1995 yields, folded with a 
stellar Initial Mass Function (IMF), lead to approximately solar abundance ratio of $\mathrm{O} / \mathrm{Fe}$ (or $\alpha$ element), without the Fe contribution of SNIa. This lead Timmes et al. (1995b) to suggest that the Fe yields of WW1995 are probably overestimated. Following their suggestion, we adopted in our model half the nominal values for the WW1995 yields for iron peak elements (from $\mathrm{Cr}-\mathrm{Zn}$ ). Taking into account the uncertainties currently affecting those yields, such a reduction is not unreasonable. Our procedure allows to produce the observed evolution of $\mathrm{O} / \mathrm{Fe}$ and $\alpha / \mathrm{Fe}$ in the Milky Way halo, and does not alter the abundance ratios between iron peak elements (which are most commonly observed in DLA systems).

To account for the additional source of Fe-peak elements, required to explain the observed decline of $\mathrm{O} / \mathrm{Fe}$ abundance ratio in the Milky Way disk (e.g. Goswami \& Prantzos 2000; GP2000), we utilise the recent yields of SNIa from the exploding Chandrashekhar-mass CO white dwarf models W7 and W70 of Iwamoto et al. (1999). These are updated versions of the original W7 model of Thielemann et al. (1986), calculated for metallicities $Z=Z_{\odot}(\mathrm{W} 7)$ and $Z=0$ (W70), respectively. These SNIa models lead to an oveproduction of $\mathrm{Ni}$, which will also be clearly seen when we compare our model results of [Ni/Zn] with observations in low redshift DLAs.

Finaly, notice that, due to uncertainties in the Zn yields of WW1995 (see Timmes et al. 1995b and PB2000a), we assume that Zn behaves always as Fe and we use then our model values of $[\mathrm{Fe} / \mathrm{H}]$ as predictions for $[\mathrm{Zn} / \mathrm{H}]$. This is justified by the fact that observations in the Milky Way disk and halo show $[\mathrm{Fe} / \mathrm{Zn}]=0$ for a large range of metallicities. However, we shall question that assumption in Sect. 4.2.

In recent papers we have shown how these metallicity dependent yields, combined with the model described in Sect. 3.1, lead to satisfactory results concerning the chemical history of the solar neighborhood (GP2000) and the current abundance gradients of intermediate mass elements in the disk of the Galaxy (Hou et al. 2000). The use of metallicity-dependent yields is a necessary condition for a consistent study of the abundance patterns in DLAs.

\subsection{Metallicity evolution vs. observations}

We calculated the evolution of the abundances of all isotopes between $\mathrm{H}$ and $\mathrm{Zn}$ in the different zones of our disk models. In this section we present results for eight elements ( $\mathrm{Zn}, \mathrm{Al}, \mathrm{Si}, \mathrm{S}, \mathrm{Mn}, \mathrm{Cr}, \mathrm{Fe}$ and $\mathrm{Ni}$ ) that have been observed in DLAs.

In Fig. 2 we present the absolute abundances of each element as a function of redshift. We display our results in two different ways. First, we show in long-dashed curves the extreme values, i.e. the highest and lowest metallicities at a given redshift, obviously corresponding to the innermost zones of massive disks and the outermost zones of low mass disks, respectively (defined in PB2000a and here to be the zones at radial distance of $0.5 R_{\mathrm{d}}$ and $5.5 R_{\mathrm{d}}$, respectively). The corresponding average value, taking into account various statistical factors (as discussed in Sect. 3.1) is given by Eq. (3) with $\Phi(\mathcal{F})=1$ in all zones and is shown by a dotted curve. It can be seen that for all elements this "unfiltered" average value, as well as the extreme values, display strong evolution and are in disagreement with the data.

The shaded area of Fig. 2 is obtained by adopting the "filter" suggested by Boissé et al. (1998) for Zn and already introduced in PB2000a, i.e. by putting in Eq. (3) $\Phi(\mathcal{F})=1$ for $18.8<\mathcal{F}<21$, and $\Phi(\mathcal{F})=0$ otherwise. The upper value of this filter $(\mathcal{F}<21)$ is maintained for all elements, since for larger values, obscuration of background quasars inhibits DLA detection (at least according to our interpretation of the observed $\mathrm{Zn} / \mathrm{H}$ vs. $N_{\mathrm{HI}}$ anticorrelation, see PB2000a). However, the lower limit $(\mathcal{F}>18)$ adopted for $\mathrm{Zn}$ corresponds to the minimal column density of $\mathrm{Zn}$ atoms detectable in current surveys; it should not be the same for other elements, since it depends (among other aspects) on which ionisation lines are used for abundance determination. We adopt then as a conservative lower limit for all other elements the condition $\log \left(N_{\mathrm{HI}}\right)>20.2$, which is simply the definition of a DLA. In other terms, we consider only the zones of our models that have surface densities $\Sigma_{\text {Gas }}>2 M_{\odot} \mathrm{pc}^{-2}$.

Applying this filter to our results (i.e. by excluding the very-metal rich and dense inner zones, as well as the low-density metal poor zones) we find a much narrower range of abundances at a given redshift, as well as a milder abundance evolution than in the original models (the solid curve in Fig. 2 gives the average value in the "filtered" zones, i.e. those with $\log \left(N_{\mathrm{HI}}\right)>20.2$ and $\mathcal{F}<21$ ). In PB2000a we found that this "biased evolution" picture is quite compatible with observations of $\mathrm{Zn}$ in DLAs. Here we show that it is also compatible with all available data on DLA abundances: indeed, both the observed dispersion at a given redshift and the mild overall evolution are nicely reproduced.

We find that in our models the average abundance varies at a rate $d<[\alpha / \mathrm{H}]>/ \mathrm{d} z \sim-0.25$ for $\alpha$-elements and $d<[\mathrm{Fe} / \mathrm{H}]>/ \mathrm{d} z \sim-0.33$ for Fe in the redshift range $z=0.5-4$. In the latter case, the larger rate is due to the introduction of a late source of Fe, namely SNIa. We note that in a recent work Savaglio et al. (1999), after correcting for depletion a large number of adundances in DLAs find that the evolution of the intrinsic DLA metallicity $Z$ as a function of redshift can be described by $\operatorname{d} \log Z / \mathrm{d} z=-0.30 \pm 0.06$ for $0.5<z<4$, i.e. a value not very different from ours.

The scatter of absolute abundances at a given redshift in our models is attributed both to the different types of galactic disks considered here (massive ones evolving more rapidly than low mass ones) and to the differential evolution of the various regions within a given disk (inner zones evolving more rapidly than the outer ones). The obtained scatter, typically a factor of 30-50 at a given redshift, reproduces fairly well the observed one. We notice that in Fig. 2 we find a smaller scatter in the "filtered" 


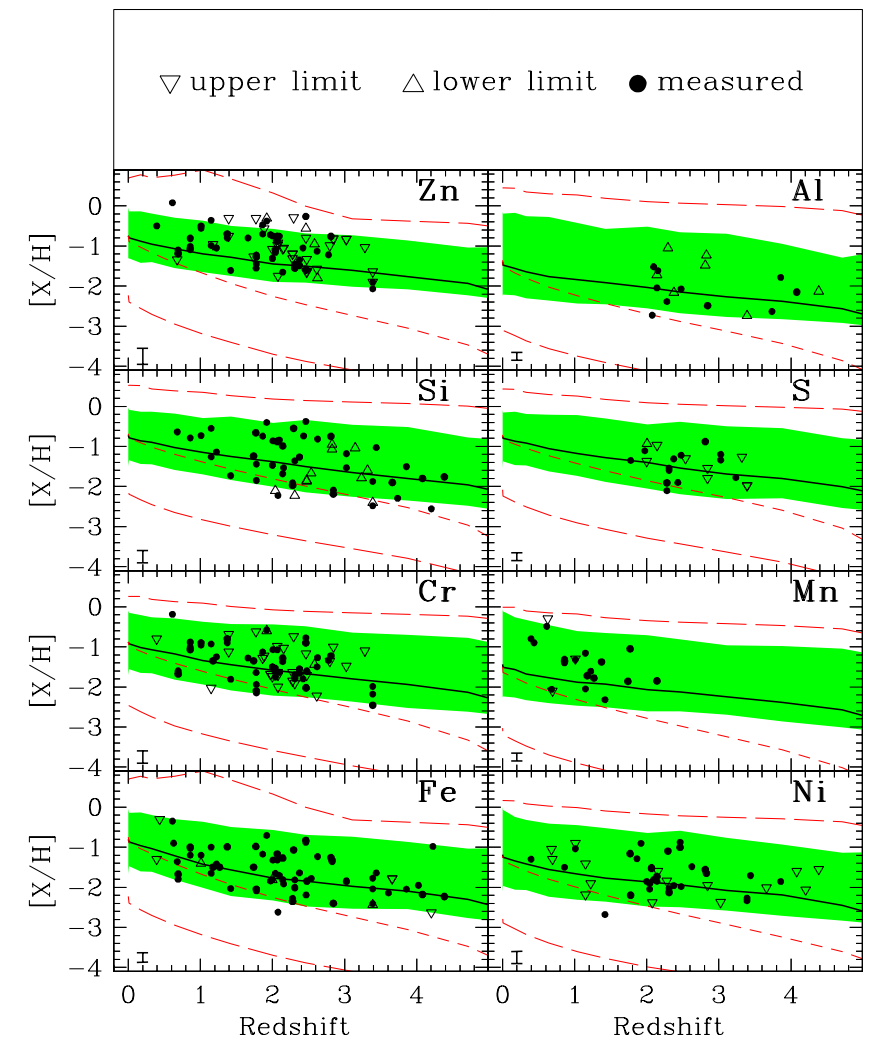

Fig. 2. Evolution of elemental abundances in our disk galaxy models for 8 elements, $\mathrm{Al}, \mathrm{Si}, \mathrm{S}, \mathrm{Cr}, \mathrm{Mn}, \mathrm{Fe}, \mathrm{Ni}$ and $\mathrm{Zn}$, as a function of redshift $z$. Observational data for DLAs are from references listed in Table 1, with typical errors shown in the bottom left corner of each panel. In each panel, the shaded area includes all the "filtered" zones of our models (i.e. by taking various biases into account, see text) and the thick curve is the corresponding mean value in those same zones. A weak evolution and an important scatter, both compatible with observations, are obtained for all elements. For comparison, we also give the results corresponding to the "unfiltered situation" (i.e. all the zones of our models): the long dashed curves correspond to the upper and lower limits, while the short dashed curve is the corresponding mean value. Our disk models (which include no correction for dust depletion effects) have a true metallicity evolution stronger than the one observed in DLAs

values of $\mathrm{Zn}$ than in those of Fe, although we assumed that both elements evolve in the same way (i.e. $[\mathrm{Zn} / \mathrm{Fe}]=$ $0)$. This is due to the different lower filters used: $\mathcal{F}>18.8$ for $\mathrm{Zn}$ and $\log (N(\mathrm{HI}))>20.2$ for Fe.

The mild evolution and the large corresponding scatter of metal abundances in DLAs have been addressed in previous works in the field. Observational papers attribute the former to selection bias (e.g. Vladilo et al. 2000) although no quantitative model is proposed to substantiate this argument. On the other hand, most theoretical papers (e.g. Timmes et al. 1996; Ferrini et al. 1997; Prantzos \& Silk 1998; Jimenez et al. 1999; Lindner et al. 1999) ignore such biases and find invariably a metallicity evolution stronger than observed. Selection biases are properly considered in PB2000a. Meusinger \& Thon (1999) also take such effects into account, but consider only disk galaxies in a narrow range of masses $\left(5-810^{10} M_{\odot}\right)$, i.e. with quite similar star formation histories. As a result, even after considering selection effects, they find that the average metallicity in their models increases by a factor of $\sim 10$ between $z=1$ and 3, much larger than suggested by the observations. For that same reason, they fail to reproduce the observed scatter at a given redshift and they obtain much smaller values.

\section{Abundance ratios and depletion patterns in DLAs}

Abundance ratios are more reliable tracers of the chemical evolution than absolute abundances. They allow one to identify whether an element originated from long-lived sources (low mass stars or SNIa) or short-lived ones (massive stars and SNII), or whether it is affected by the oddeven effect. The analysis of abundance ratios in the Milky Way disk and halo stars provides important insight on the chemical history of the Milky Way as well as on the different production sites of various elements (despite the existence of large uncertainties in both theory and observations, see GP2000).

However, situations in DLAs are more complicated. The abundances observed in DLAs may not represent the real chemical composition of the system if part of the elements is removed from the gas to the solid phase (dust grains), as happens in the interstellar medium of our Galaxy. In the discussion of Sect. 3.3, we did not consider any such kind of dust depletion effects. We have argued, in Sect. 2, that the observed abundance ratios $[\mathrm{X} / \mathrm{Zn}]$ in DLAs, when plotted as a function of $\mathcal{F}$, suggest that some depletion does occur in those systems, at least for $\mathrm{Fe}, \mathrm{Cr}$, $\mathrm{Mn}$ and Ni. We have also argued that the depletion pattern for Fe, when compared to the one observed in the Milky Way, suggests that the line of sight intercepts several gas clouds in a given DLA.

In this section, we compare observed abundance patterns in DLAs with those obtained in our models of disk galaxy evolution. Our aim is to find out: i) whether there are any discrepancies between models and observations, ii) whether such discrepancies can be attributed to depletion, and iii) whether the resulting depletion patterns correspond to well known ones, such as those observed in the local disk or halo clouds.

\subsection{Abundance ratios in DLAs}

In Fig. 3 we plot the evolution of abundance ratios $[\mathrm{X} / \mathrm{Zn}]$ as a function of metallicity $(=[\mathrm{Zn} / \mathrm{H}])$, redshift and metal column density $(\mathcal{F})$ for the elements $\mathrm{Al}, \mathrm{Si}, \mathrm{S}, \mathrm{Cr}, \mathrm{Mn}, \mathrm{Fe}$ and $\mathrm{Ni}$. As in many other works, we assume that $\mathrm{Zn}$ is not depleted at all in dust and, therefore, it can be used as a tracer of metallicity. As explained in Sect. 3.2, because of uncertainties about the nucleosynthesis of $\mathrm{Zn}$, we assume that $[\mathrm{Fe} / \mathrm{Zn}]=0$ always in our model. The heavy shaded regions in Fig. 3 present the results corresponding to the "filtered" zones of our models (i.e. satisfying DLA 


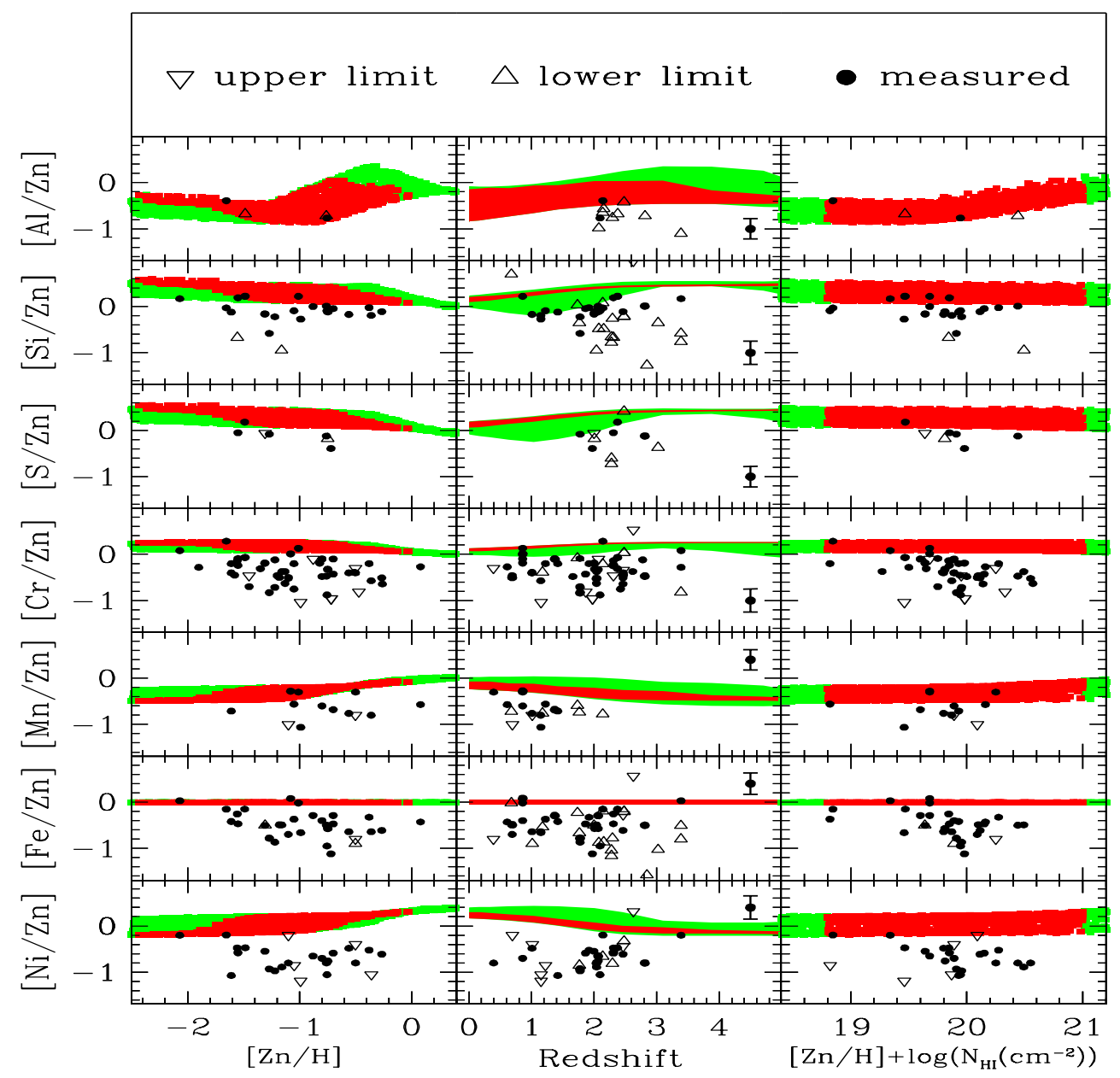

Fig. 3. Abundance ratios versus metallicity (left) redshift (middle) and metal column density $\mathcal{F}$ (right). In all panels, the light shaded area includes all the zones of our models, while the heavy shaded area only those corresponding to the "filtered" zones (i.e. satisfying DLA constraints, see Sect. 3.3). Note that $[\mathrm{Fe} / \mathrm{Zn}]=0$ in our models, because we have assumed that $[\mathrm{Zn} / \mathrm{H}]=[\mathrm{Fe} / \mathrm{H}]$ always (see Sect. 3.2). Data for DLAs are from Table 1 and typical error bars are displayed in the middle panels. Deviation of the data from the model values at a given redshift is, in principle, attributed to dust depletion, which is not included in the models (see discussion in Sect. 4.2)

constraints), while the light shaded ones correspond to all other zones.

$\alpha$ ELEMENTS Si and S: $\alpha$ elements present a well known behaviour in our Milky Way disk and halo. The $[\alpha / \mathrm{Fe}]$ ratio is nearly constant in the halo, at a value of $\sim 0.4$, and declines gradually in the disk from $[\mathrm{Fe} / \mathrm{H}] \sim-1$ to 0 . The latter feature is attributed to the late $(\sim 1 \mathrm{Gyr})$ contribution of SNIa to the disk composition.

The observed $[\mathrm{Si} / \mathrm{Zn}]$ ratios in DLAs do not show any trend with metallicity, redshift or column density, and they are solar, on average (taking into account observational uncertainties). Our model results, when plotted as a function of metallicity or column density cannot be directly compared with the corresponding data, because a given metallicity in the model may be reached at widely different epochs, dependent on the chemical history of the corresponding region. Only when $[\mathrm{X} / \mathrm{Zn}]$ is plotted as a function of redshift can a meaningful comparison to the data be made. From the middle panels of Fig. 3, it can be seen that at early times $(z>2.5)$ the model $[\mathrm{Si} / \mathrm{Zn}] \sim$ 0.4 , with a very small variation around that value.

At late times $[\mathrm{Si} / \mathrm{Zn}]$ declines in general, albeit with large variations. The decline is due to the late appearence of Fe-producing SNIa and to our assumption that $[\mathrm{Fe} / \mathrm{Zn}]=0$ always. There are large variations of [Si/Zn] between the various zones of our models (light shaded area), because SNIa enter the scene at different times depending on the previous SFR history of the corresponding zones. In the "filtered" zones, however, the scatter around the mean value is very small. Compared to that well defined model trend, the data show systematically lower values. This difference could, in principle, be attributed to depletion by $\sim 0.4 \mathrm{dex}$, an amount comparable with the one seen in the local warm clouds of the disk and the halo.

However, $[\mathrm{S} / \mathrm{Zn}]$ in our models behaves exactly as [Si $/ \mathrm{Zn}]$ (for the same reasons) and the corresponding DLA data suggest again the same degree of depletion. This 
is difficult to understand, since $\mathrm{S}$ is not expected to be depleted at all. Ockam's razor, along with the fact that $\mathrm{S} / \mathrm{Zn}$ and $\mathrm{Si} / \mathrm{Zn}$ in DLAs are independent of Zn column density (Fig. 1) suggest then that these two elements are not depleted in DLAs; they also suggest that the intrinsic DLA ratios of those elements are $\sim$ solar, irrespectively of redshift.

This conclusion does not answer the question about the nature of DLAs. Several authors (Centurion et al. 2000; Jimenez et al. 1999; Vladilo 1999) suggested that Low Surface Brightness galaxies (LSBs) are good candidates, since they probably have different nucleosynthetic histories (at present unknown and unconstrained). At this point we emphasize that our scenario involves many different chemical histories: disks of different masses form the bulk of their stars at different epochs and within them different zones evolve at different rates. As a result, at late times, the $[\alpha / \mathrm{Zn}]$ ratio varies considerably in our models. But the zones satisfying the DLA criteria $(\mathcal{F}>18.8$ for $\mathrm{Zn}$ and $\log (N(\mathrm{HI}))>20.2$ for other elements) display a narrow range of values at all times. We shall return to that point in Sect. 4.2.

Fe PEAK Elements: Cr, Mn, Fe and Ni are produced by massive stars in the early life of the Milky Way and mostly by SNIa at late times. Observations of their abundances in halo and disk stars show that $\mathrm{Cr}, \mathrm{Fe}$ and $\mathrm{Ni}$ behave similarly, at least down to $[\mathrm{Fe} / \mathrm{H}] \sim-2$, while the monoisotopic $\mathrm{Mn}$ behaves differently: the $[\mathrm{Mn} / \mathrm{Fe}]$ ratio is $\sim-0.4$ in the halo and increases to its solar value in the disk. This behaviour is well accounted for by the metallicity dependent yields of WW1995 for massive stars and those of Iwamoto et al. (1999) for SNIa, as shown by detailed chemical evolution models in GP2000.

Our models show that the $[\mathrm{Cr} / \mathrm{Zn}]$ and (by assumption) $[\mathrm{Fe} / \mathrm{Zn}]$ ratios are $\sim$ solar and independent of metallicity or redshift. As expected, $[\mathrm{Mn} / \mathrm{Fe}] \sim-0.4$ at low metallicities (high redshifts) and increases slowly at late epochs. The small overproduction obtained for [Ni/Zn] obtained at late times has a well known origin: the adopted SNIa yields of Iwamoto et al. (1999) which oveproduce Ni by a factor of $\sim 2$ (see GP2000 for a discussion). Taking $[\mathrm{Ni} / \mathrm{Zn}]=0$ over the whole metallicity range would thus be more appropriate.

In all four cases, the observed $[\mathrm{X} / \mathrm{Zn}]$ ratio in DLAs is clearly below solar or model values, pointing to depletion as the most obvious explanation. This conclusion is corroborated by the observed anticorrelation of $[\mathrm{X} / \mathrm{Zn}]$ with Zn column density, as argued in Sect. 2.

In a recent work Pettini et al. (2000) selected a small subsample of $[\mathrm{Mn} / \mathrm{Fe}]$ values in DLAs with $[\mathrm{Zn} / \mathrm{Cr}]<0.3$, i.e. with small amount of depletion; assuming then that all Fe peak elements are depleted by the same (small) amount, they found that $[\mathrm{Mn} / \mathrm{Fe}] \sim-0.4$ in DLAs, independent of metallicity. This picture is not quite compatible with the one in the Milky Way, but because of their small sample it is difficult to conclude whether their finding has implications for the role of SNIa in DLAs or not.

\subsection{Depletion patterns}

From the discussion of the previous section it turns out that several elements in DLAs are depleted, at least with respect to the predictions of our chemical evolution models, which reproduce satisfactory several DLA features (e.g. Fig. 2). It is interesting to see how the predicted depletion patterns compare to some well known ones, as those of the warm halo and disk clouds in the Milky Way.

In the upper panels of Fig. 4, we compare the observed $[\mathrm{X} / \mathrm{Zn}]$ ratios in DLAs to the corresponding ones of our models i.e. at the absorbers' redshifts, (as given in the middle panels of Fig. 3). Taking into account the discussion of Sect. 2, namely the fact that the depletion patterns depend on $\mathrm{Zn}$ column density, we split the data into low and high $\mathcal{F}$ values $(\mathcal{F}<19.8$ and $\mathcal{F} \geq 19.8$, respectively). We also show, for comparison (in both panels) the depletion patterns in the Milky Way warm halo and disk clouds (open symbols, connected by solid lines, data from Savage $\&$ Sembach 1996). Due to the uncertainties (unknown intrinsic abundances in DLAs, measurement errors), such a comparison has no sense for individual DLAs, but only in a statistical sense.

In the left upper panel of Fig. 4, it is seen that the depletion amount obtained at low $\mathcal{F}$ is, on average, smaller than in warm halo clouds. The average depletion is similar for all elements, $\sim 0.2$ dex, and comparable to the error bars of the measurements. In the right upper panel of Fig. 4, the depletion is slightly larger for all elements. The depletion pattern ressembles, on average, the one of warm halo clouds for $\mathrm{Fe}, \mathrm{Ni}$ and $\mathrm{Cr}$, but also for $\mathrm{Mn}$ and $\mathrm{Si}$ (i.e. within error bars). However, as dicussed in the previous section, it is simply impossible to accomodate $\mathrm{S}$ in this scheme, since it is hard to imagine that its ratio to $\mathrm{Zn}$ can be subsolar.

The problem with S may be due to the fact that we use as a reference pattern the one resulting from our models. We checked then whether using the solar abundance ratios as the reference pattern could lead to a better fit of the data. The results of that comparison appear in the lower panels of Fig. 4, again for low and high $\mathcal{F}$ values (left and right lower panels, respectively). At low $\mathcal{F}$ hardly any depletion at all is found, for all elements except Mn; this is puzzling, since Mn cannot be more depleted than the other Fe peak elements. At high $\mathcal{F}$, the resulting depletion pattern compares fairly well with the one of the warm halo clouds for all elements.

In summary: if one assumes that the intrinsic metallicity pattern in DLAs is the one given by our models, a difficulty arises with $\mathrm{S}$, which is found to be depleted at high $\mathcal{F}$. If, on the other hand, it is assumed that the intrinsic DLA pattern is solar, a problem arises with Mn, which is found to be depleted at low $\mathcal{F}$.

We note that our assumption of $[\mathrm{Fe} / \mathrm{Zn}]=0$ always, although based on observations of Milky Way stars, may be inconsistent and potentially wrong. Indeed, it implies that $\sim 2 / 3$ of $\mathrm{Zn}$ are produced at late times by SNIa, exactly as is Fe in the solar vicinity (see GP2000). This 


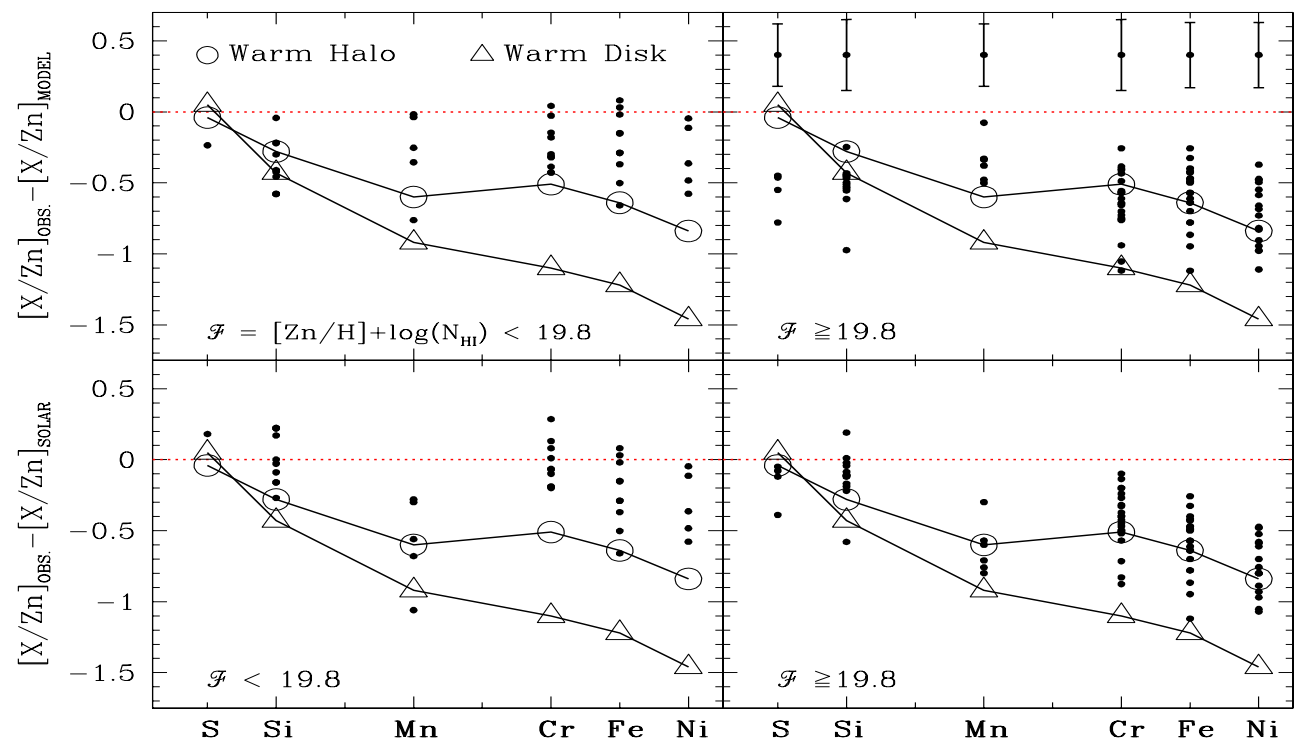

Fig. 4. Depletion patterns in DLAs. In the upper panels, observed DLA abundance ratios $[\mathrm{X} / \mathrm{Zn}]$ are compared to those resulting from our models, taken at the absorber's redshift. In the lower panels, the intrinsic DLA abundance pattern is simply assumed to be solar. In both cases, the DLA data are plotted according to the corresponding Zn column density, given by $\mathcal{F}=[\mathrm{Zn} / \mathrm{H}]+$ $\log \left(N_{\mathrm{HI}}\right)$ : left panels correspond to $\mathcal{F}<19.8$ and right panels to $\mathcal{F} \geq 19.8$. In all panels the depletion patterns obtained in warm halo and disk clouds in the Milky Way are shown by open symbols, connected with solid lines (data from Savage \& Sembach 1996). Typical error bars for measured $[\mathrm{X} / \mathrm{Zn}]$ ratios in DLAs are displayed in the right upper panel

assumption introduces a time-delay in the production of the bulk of $\mathrm{Zn}$ with respect to the one of other $\alpha$-elements, like $\mathrm{S}$, coming from massive stars. The reason for that delay is that the bulk of Fe is always produced with some delay in our models, through SNIa explosions. This explains the obtained decline of $\alpha / \mathrm{Zn}$ in our models only at redshifts lower than $\sim 2$. However, physically, this picture is not correct: it is well known that SNIa do not produce Zn (e.g. Iwamoto et al. 1999). The fact that in the solar vicinity $\mathrm{Zn}$ behaves as $\mathrm{Fe}$ at late times also is obviously a coincidence. The late Fe production of SNIa is matched by the $\mathrm{Zn}$ production of SNII, which is metallicity-dependent in both the yields of WW1995 as well as in the recent work of Limongi et al. (2000). The yields of WW1995, however, do not reproduce the observed evolution of $\mathrm{Zn} / \mathrm{Fe}$ in the Milky Way and we used Fe as a proxy for Zn; in that way we "forced" a pure metallicity effect (which can, in principle, manifest quite early in zones that evolve rapidly to high metallicities) to mimic a time-delay effect. In that sense, our treatment of $\mathrm{Zn}$ is not quite correct. Obviously, the nucleosynthesis of $\mathrm{Zn}$ should be carefully scrutinised in future models of massive star nucleosynthesis.

At this point it seems difficult to gain more insight into the nature of DLAs by simply considering their abundance patterns. We think, however, that progress can be made through further observations of $\mathrm{S}$ in DLAs of high Zn column density and of Mn in DLAs of low Zn column density; such observations would certainly help to establish whether the aforementioned trends are real or not.

\section{Summary}

In this work, we study the chemical abundance patterns observed in DLAs. First, we show that there is strong observational evidence for depletion of the $\mathrm{Fe}$-peak elements, obtained when their abundance ratios $[\mathrm{X} / \mathrm{Zn}]$ are plotted as a function of $\mathrm{Zn}$ column density. The resulting anticorrelation is reminiscent of similar depletion patterns obtained in Milky Way clouds as a function of gas column density. However, the intrinsic gas metallicity does not differ much from solar in Milky Way clouds, while it varies by factors $\sim 100$ in DLAs. For that reason we argue that $Z n$ column density is a more appropriate parameter to measure depletion, since it is proportional (to a first approximation) to the amount of dust along the line of sight. Further measurements of $[\mathrm{X} / \mathrm{Zn}]$ ratios as a function of $\mathcal{F}=[\mathrm{Zn} / \mathrm{H}]+\log \left(N_{\mathrm{HI}}\right)$ in DLAs will help to put the anticorrelation found here on a firmer basis.

Assuming that DLAs are (proto-)galactic disks, we study their chemical evolution with detailed models, making use of metallicity-dependent yields from massive stars and SNIa; these models reproduce quite successfully a large number of properties of spirals at low and high redshift as shown in a series of papers (Boissier et al. 2001; BP2000, 1999; PB2000a, 2000b). By taking into account observational constraints on the gas and metal column densities of DLAs (namely that $\log \left(N_{\mathrm{HI}}>20.2\right.$ and $18.8<\mathcal{F}<21$ ) we find that our models reproduce fairly well (and without including any depletion effects) the observed weak evolution of DLA metallicity with redshift and the corresponding scatter, for several elements detected in DLAs: S, Si, Mn, Cr, Fe, Ni and Zn. 
Encouraged by the success of the model, we studied the corresponding predicted abundance patterns. We find no clear correlation of $[\mathrm{X} / \mathrm{Zn}]$ with metallicity or $\mathrm{HI}$ column density. We also find that $\mathrm{Si} / \mathrm{Zn}$ and $\mathrm{S} / \mathrm{Zn}$ are supersolar at high redshifts, while $\mathrm{Mn} / \mathrm{Zn}$ is subsolar. The latter effect results from the metallicity dependent yields adopted here and is also observed in the Milky Way (see GP2000 and references therein). The former results from our assumption that $[\mathrm{Zn} / \mathrm{Fe}]=0$ always, an assumption based on observations in the Milky Way and made because of the poor understanding of Zn nucleosynthesis.

Comparing the observations of $[\mathrm{X} / \mathrm{Zn}]$ in DLAs to our model calculations (which do not include the effects of dust depletion), we find evidence for dust depletion effects in the observations for $\mathrm{Cr}, \mathrm{Ni}, \mathrm{Fe}, \mathrm{Mn}$ and $\mathrm{Si}$, for high $\mathcal{F}>19.8$. This depletion is $\sim 0.4$ dex, on average, and comparable to the one observed in warm halo clouds. However, a difficulty arises with $\mathrm{S}$ which is found to be depleted at high $\mathcal{F}$. If on the other hand it is assumed that the intrinsic DLA pattern is solar, a problem arises with $\mathrm{Mn}$, which is found to be depleted at low $\mathcal{F}$.

We think that currently-available data on DLA abundances do not allow us to establish the intrinsic abundance patterns of those systems. Progress can be made through further observations of abundances in DLAs, in particular those of $\mathrm{Mn}$ and S, by taking into account the aforementionned role of the metal column density. We also note that the conclusions of this work are largely based on the, presently poorly understood, nucleosynthesis of $\mathrm{Zn}$; the production of that element should be carefully studied in new models of massive star nucleosynthesis.

Acknowledgements. J. L. Hou acknowledges the warm hospitality of the IAP (Paris, France). We are grateful to Patrick Petitjean for useful discussions and comments on this subject. This work was made possible thanks to the support of China Scholarship Council (CSC) and National Natural Sciences Foundation of China and the NKBRSF19990754.

\section{References}

Boissé, P., Le Brun, V., Bergeron, J., \& Deharveng, J. M. 1998, A\&A, 333, 841

Boissier, S., Boselli, A., Prantzos, N., \& Gavazzi, G. 2001, MNRAS, in press [astro-ph/0009232]

Boissier, S., \& Prantzos, N. 2000, MNRAS, 312, 398 (BP2000)

Boissier, S., \& Prantzos, N. 1999, MNRAS, 307, 857 (BP1999)

Centurion, M., Bonifacio, P., Molaro, P., \& Vladilo, G. 2000, ApJ, 536, 540

Centurion, M., Bonifacio, P., Molaro, P., \& Vladilo, G. 1998, ApJ, 509, 620

de la Varga, A., Reimers, D., Tytler, D., Barlow, T., \& Burles, S. 2000 [astro-ph/0008366]

Ferrini, F., Molla, M., \& Diaz, A. I. 1997, ApJL, 487, 29

Gonzalez, A., Williams, K., Bullock, J., Kolatt, T. S., \& Primack, J. R. 2000, ApJ, 528, 145

Goswami, A., \& Prantzos, N. 2000, A\&A, 359, 191 (GP2000)

Haehnelt, M., Steinmetz, M., \& Rausch, M. 1998, ApJ, 495, 647
Howk, J. C., \& Sembach, K. 1999, ApJ, 523, L141

Hou, J. L., Prantzos, N., \& Boissier, S. 2000, A\&A, 362, 921

Izotov, Y., Scherrer, D., \& Charbonnel, C. 2000, AA, in press [astro-ph/0010643]

Iwamoto, K., Brachwitz, F., Nomoto, K., et al. 1999, ApJS, 125,439

Jimenez, R., Bowen, D. V., \& Matteucci, F. 1999, ApJ, 514, L83

Jimenez, R., Padoan, P., Matteucci, F., \& Heavens, A. 1998, MNRAS, 299, 123

Lauroesch, J. T., Truran, J. W., Welty, D. E., \& York, D. G. 1996, PASP, 108, 641

Lindner, U., von-Alvensleben, U. F., \& Fricke, K. J. 1999, A\&A, 341, 709

Lu, L., Sargent, W. L. W., Barlow, T. A., Churchill, C. W., \& Vogt, S. S. 1996, ApJS, 107, 475

Matteucci, F., Molaro, P., \& Vladilo, G. 1997, A\&A, 321, 45

Meusinger, H., \& Thon, R. 1999, A\&A, 351, 841

Mo, H. J., Mao, S. D., \& White, S. D. M. 1998, MNRAS, 295, 319

Molaro, P., Bonifacio, P., Centurion, M., et al. 2000, ApJ, 541, 54

Molaro, P., Centurion, M., \& Vladilo, G. 1998, MNRAS, 293, L37

Pei, Y. C., \& Fall, S. M. 1995, ApJ, 454, 69

Petitjean, P., \& Ledoux, C. 1999, Ap\&SS, 265, 483

Pettini, M., Ellison, S. L., Steidel, C., Shapley, A. E., \& Bowen, D. V. 2000, ApJ, 532, 65

Pettini, M., Ellison, S. L., Steidel, C. C., \& Bowen, D. V. 1999, ApJ, 510, 576

Pettini, M., King, D. L., Smith, L.J., \& Hunstead, R. W. 1997a, ApJ, 478, 536

Pettini, M., Smith, L. J., King, D. L., \& Hunstead, R. W. 1997b, ApJ, 486, 665

Pettini, M., Smith, L. J., Hunstead, R. W., \& King, D. L. 1994, ApJ, 426, 79

Prantzos, N., \& Boissier, S. 2000a, MNRAS, 315, 82 (PB2000a)

Prantzos, N., \& Boissier, S. 2000b, MNRAS, 313, 338 (PB2000b)

Prantzos, N., \& Silk, J. 1998, ApJ, 507, 229

Prochaska, J. X., \& Wolfe, A. M. 2000, ApJ, 533, L5

Prochaska, J. X., \& Wolfe, A. M. 1999, ApJS, 121, 369

Savage, B. D., \& Sembach, K. R. 1996, ARA\&A, 34, 279

Savaglio, S., Panagia, N., \& Stiavelli, M. 1999, in Cosmic Evolution and Galaxy Formation, ed. J. Franco et al., in press [astro-ph/9912112]

Thielemann, F., Nomoto, K., \& Yokoi, K. 1986, A\&A, 158, 17

Timmes, F. X., Lauroesch, J. T., \& Truran, J. W. 1995a, ApJ, 451,468

Timmes, F. X., Woosley, S. E., \& Weaver, T. A. 1995b, ApJS, 98,617

Valageas, P., Schaeffer, R., \& Silk, J. 1999, A\&A, 691, 711

Vladilo, G., Bonifacio, P., Centurion, M., \& Molaro, P. 2000, ApJ, in press [astro-ph/0005555]

Vladilo, G. 1999, in The evolution of galaxies on cosmological timescales, ed. J. E. Beckman, \& T. J. Mahoney, ASP Conf. Ser., 187, 323

Vladilo, G. 1998, ApJ, 493, 583

Wakker, B. P., \& Mathis, J. S. 2000, ApJL, accepted [astro-ph/0010045]

Woosley, S. E., \& Weaver, T. A. 1995, ApJS, 101, 181 (WW1995) 